\title{
Presynaptic Local Signaling by a Canonical Wingless Pathway Regulates Development of the Drosophila Neuromuscular Junction
}

\author{
Claudia Miech, ${ }^{1}$ Hans-Ulrich Pauer, ${ }^{2} \mathrm{Xi} \mathrm{He},{ }^{1}$ and Thomas L. Schwarz ${ }^{1,3}$ \\ ${ }^{1}$ F. M. Kirby Neurobiology Center, Children's Hospital, Boston, Massachusetts 02115, ${ }^{2}$ Division of Hemostasis and Thrombosis, Department of Medicine, \\ Beth Israel Deaconess Medical Center and Harvard Medical School, Boston, Massachusetts 02215, and ${ }^{3}$ Department of Neurobiology, Harvard Medical \\ School, Boston, Massachusetts 02115
}

\begin{abstract}
Wnt/wingless signaling contributes to the development of neuronal synapses, including the Drosophila neuromuscular junction. Loss of $w g$ (wingless) function alters the number and structure of boutons at this model synapse. Examining Wnt/wingless signaling mechanisms, we find that a distinct pathway operates presynaptically in the motoneuron and can account for many of the effects of wingless at this synapse. This pathway includes the canonical elements arrow/LRP (low-density lipoprotein receptor-related protein), dishevelled, and the glycogen synthase kinase shaggy (GSK3) and regulates the formation of microtubule loops within synaptic boutons as well as the number of synaptic boutons. This pathway, however, appears to be independent of $\beta$-catenin signaling and the transcriptional regulation that is most frequently downstream of these components. Instead, inhibition of shaggy is likely to act locally. This pathway thus provides a parallel mechanism to the postsynaptic activation of frizzled receptors and indicates that synaptic development results from the bidirectional influence of wingless on both presynaptic and postsynaptic structures via distinct intracellular pathways.
\end{abstract}

Key words: canonical wingless signaling; synaptic; shaggy; futsch; Drosophila; neuromuscular junction

\section{Introduction}

The development of synapses is regulated by membrane proteins (Scheiffele et al., 2000; Dean and Dresbach, 2006) and secreted molecules (Hall et al., 2000; Packard et al., 2002, 2003). The latter include the Wnt/wingless (wg) morphogens, which have been implicated in the development of cerebellar synapses (Hall et al., 2000) and Drosophila neuromuscular junctions (Packard et al., 2002 and Packard et al., 2003). Pathways activated by Wnt/wingless have been studied primarily in the context of patterning, cell polarity, and cancer, but less is known about their intracellular mechanisms at synapses.

Multiple Wnt pathways have been described (Logan and Nusse, 2004), including the canonical transcriptional regulation pathway mediated by $\beta$-catenin, a local and cytoplasmic variant of the canonical pathway (Ciani et al., 2004), the planar cell polarity pathway (Axelrod et al., 1998), and a pathway entailing the nuclear translocation of the cleaved fz (frizzled) receptor

Received Aug. 20, 2008; accepted Sept. 4, 2008.

This work was supported by the German Research Foundation (Deutsche Forschungsgemeinschaft) Postdoctoral Fellowship (C.M.), Leukemia and Lymphoma Society Scholarship (X.H.), and National Institutes of Health Grants MH075058 and NS041062 (T.L.S.). We thank Roel Nusse, Steve DiNardo, Kevin Moses, and Jeffrey Axelrod for sending fly stocks and Vivian Budnik, Steve DiNardo, Aaron DiAntonio, and Kyung-0k Cho for reagents. We are grateful to Matthew Salanga, Thomas Diefenbach, Lihong Bu, and the Developmental Disorders Research Center imaging core for assistance with confocal microscopy and to Timothy Mosca, Eve Pogoda, and John Salogiannis for experimental assistance.

Correspondence should be addressed to Thomas L. Schwarz, Department of Neurobiology, Harvard Medical School, 300 Longwood Avenue, Boston, MA 02115. E-mail: thomas.schwarz@childrens.harvard.edu. DOI:10.1523/JNEUROSCI.0164-08.2008

Copyright $\odot 2008$ Society for Neuroscience ～0270-6474/08/2810875-10\$15.00/0
(Mathew et al., 2005). The most extensively studied is the canonical pathway that entails transcriptional regulation by $\beta$-catenin [armadillo (arm) in Drosophila]. In this pathway, Wnt/wingless ligands interact with Frizzled receptors and their likely coreceptor, LRP5/6 [low-density lipoprotein receptor-related protein 5 and 6; arrow (arr) in Drosophila] (Pinson et al., 2000; Tamai et al., 2000, Wehrli et al., 2000) (for review, see He et al., 2004). These receptors together with the cytoplasmic phosphoprotein Dishevelled (Moon, 2005; Wallingford and Habas, 2006) stabilize $\beta$-catenin via inhibition of the $\beta$-catenin destruction complex. Inhibition of the complex prevents the phosphorylation of $\beta$-catenin by glycogen synthase kinase $3 \beta$ (GSK3 $\beta$; shaggy in Drosophila), and hypophosphorylated $\beta$-catenin translocates to the nucleus.

Although all the elements of this canonical pathway have been observed in neurons, it is not clear whether this pathway regulates synapse formation. Indeed, a variant of this pathway occurs in cerebellar nerve endings (Hall et al., 2000; Krylova et al., 2000; Ciani et al., 2004; Ahmad-Annuar et al., 2006), where the molecules of the canonical pathway inhibit GSK3 $\beta$ activity but regulate development independently of nuclear $\beta$-catenin and gene regulation. Instead, local actions of Dishevelled and GSK3 $\beta$ influence the assembly of presynaptic proteins and microtubule structure, including the phosphorylation state of the microtubule-associated protein MAP1B (Goold et al., 1999; Gordon-Weeks et al., 2000; Ciani et al., 2004; Trivedi et al., 2005; Ahmad-Annuar et al., 2006). The planar cell polarity pathway, in contrast, has not yet been shown to mediate Wnt/wg function at 
synapses, although components of the pathway, such as RhoA and Rac, are known to influence dendrite formation (Elia et al., 2006).

At the Drosophila neuromuscular junction, a novel wingless function has been uncovered (Packard et al., 2002; Mathew et al., 2005; Ataman et al., 2006) in which the C terminus of postsynaptic frizzled 2 is cleaved and thereupon translocates to the muscle nucleus. The presence of these postsynaptic events does not preclude additional signaling pathways in the presynaptic nerve endings (Speese and Budnik, 2007). Indeed, the Drosophila GSK $3 \beta$ homolog shaggy can promote growth and differentiation at this synapse by acting presynaptically (Franco et al., 2004), an observation suggesting that wingless may also act via shaggy.

In the present study, we demonstrate that wingless directly signals to the presynaptic endings at the Drosophila neuromuscular junction (NMJ), where it activates components of the canonical pathway and, bypassing transcriptional control, locally regulates microtubules. Inhibiting this presynaptic pathway reduces bouton growth and synapse differentiation, mimicking the phenotype of $w g$.

\section{Materials and Methods}

Genetics. Flies were raised on standard media at $25^{\circ} \mathrm{C}$, except as indicated. $w g^{t s(I-12)} \quad$ (FBst0007000), arr $^{2}$ (FBst0003087), UAS-sgg $g^{A 81 T}$ (FBst0005360), UAS-arm ${ }^{\text {S1O }}$ (FBti0012489), and UAS-pan ${ }^{\Delta N}$ (FBst0004784) flies were from the Bloomington Drosophila Stock Center, $w g^{C X 4}$ from Roel Nusse (Stanford University, Stanford, CA), and $\operatorname{arr}^{K 08131}$ and UAS-arr from Steve DiNardo (University of Pennsylvania, Philadelphia, PA). UAS-wg was obtained from Kevin Moses (Janelia Farms, Ashburn, VA), UAS-mitoGFP (Pilling et al., 2006) from William Saxton (University of California, Santa Cruz, CA), and UAS-mCD 8 was obtained from Liquin Luo (Stanford University, Stanford, CA) (Lee and Luo, 1999). The UAS- $d s h^{D I X}$ and the green fluorescent protein (GFP)tagged full-length dishevelled $(d s h)$ were from Jeffrey Axelrod (Axelrod et al., 1998). Flies were outcrossed to the pan-neuronal elav-Gal4 driver (Yao and White, 1994), the muscle-specific MHC-Gal4 driver, or the glia-specific repo-GAL4 driver. The wild-type (wt) flies were $w^{1118}$. For temperature pulses in $w^{C X 4} / w^{t s}$, larvae were raised at $17^{\circ} \mathrm{C}$ and shifted to $29^{\circ} \mathrm{C}$ (the restrictive temperature) for $18 \mathrm{~h}$ at early third-instar larval stages.

Immunohistochemistry. Body wall muscles from wandering thirdinstar larvae were dissected in $\mathrm{Ca}^{2+}$-free HL-3 medium (Stewart et al., 1994) and fixed in Bouin's fixative for $90 \mathrm{~s}$ or, for localization of armadillo, shaggy, or arrow, in $4 \%$ formaldehyde (as paraformaldehyde) for 8 min. Larvae were then washed with PBS containing $0.1 \%$ Triton X-100 (PBT) and blocked in 5\% normal goat serum (NGS) in PBT for $1 \mathrm{~h}$. Blocking was followed by incubation in primary antibodies in $5 \%$ NGS/ PBT for at least $2 \mathrm{~h}$. After five washes in PBT for a total of $2 \mathrm{~h}$, the larvae were incubated in secondary antibodies in 5\% NGS/PBT for $1.5 \mathrm{~h}$ and washed five times in PBT and twice in PBS. Larvae were mounted on slides in Vectashield (Vector Laboratories). The following primary antibodies were used: rabbit polyclonal anti-wg at 1:200 (Reichsman et al., 1996), rabbit polyclonal anti-arr, preabsorbed against wild-type embryos at 1:5000 (Rives et al., 2006), rabbit polyclonal anti-GluRIIC at 1:2000 (Marrus et al., 2004), rabbit polyclonal anti-discs large (Dlg) at 1:1000 (Cho et al., 1992), mouse monoclonal anti-GSK3 at 1:100 (Millipore), mouse monoclonal anti-tubulin at 1:400 (Sigma-Aldrich), and FITC- or Cy5-conjugated goat anti-horseradish peroxidase (HRP) at 1:100 (Jackson ImmunoResearch). From the Developmental Studies Hybridoma Bank, University of Iowa, we obtained the following mouse monoclonal antibodies: anti-arm (N27A1), used at 1:50, anti-bruchpilot (nc82) at 1:100 (E. Buchner, Universitaet Wuerzburg, Wuerzburg, Germany), anti-futsch $(22 \mathrm{C} 10)$ at $1: 50$, and anti-Dlg (4FC) at 1:500. DAPI $\left(4^{\prime}, 6^{\prime}-\right.$ diamidino-2-phenylindole) was used for DNA counterstaining (Vectashield). The following secondary antibodies were used: FITC-, Cy3-, and Cy5-conjugated goat anti-mouse at 1:100, 1:400, and 1:1000; and Cy3- and Cy5-conjugated donkey anti-rabbit at 1:200 (Jackson ImmunoResearch Laboratories).

Microscopy and morphological quantification. Fixed and labeled larvae were imaged with a Zeiss LSM510 confocal microscope with a 63/1.4 objective. Single high-resolution images were acquired as stacks of multitracked images with separate channels and processed with the LSM software or Adobe Photoshop and Adobe Illustrator. Fluorescent images for quantification assays were captured with a Nikon microscope (Eclipse E800) using Spot advanced image software (version 3.4.4). For comparison between genotypes, samples were processed simultaneously and imaged using identical microscopic acquisition parameters. For the quantification of type I boutons and futsch distribution, body wall muscle preparations were double stained with anti-HRP and anti-futsch. Satellites were identified and quantified according to Estes et al. (2003). Any single bouton that was not included in a chain of boutons, but instead appeared to be a lateral sprout, was counted as a satellite bouton. The experimenter was blinded to genotypes during both imaging and analysis.

Statistics. Statistical analysis was performed using ANOVA to compare different genotypes to a wild-type control within an experimental group, followed by Dunnett's post hoc test. Error bars are SD.

\section{Results}

The canonical wg pathway proteins wg, arrow, dishevelled, and shaggy localize to the synapses of Drosophila NMJs

To ask which molecules may transduce the extracellular wingless signal at the NMJ of third-instar Drosophila larvae, we performed immunocytochemistry with antibodies specific for known components of the wingless signaling pathway: wingless, arrow, shaggy, and armadillo (Fig. 1). We costained with antibodies against HRP, to label neuronal membranes (Jan and Jan, 1982). To label postsynaptic specializations, we used antibodies against the postsynaptic density protein Dlg, a Drosophila homolog of the mammalian PSD-95.

As expected from previous studies (Packard et al., 2002), we observed wingless immunoreactivity to be concentrated at synapses at the NMJ, although punctate staining in muscle cytoplasm was also seen away from the synapse. The staining appeared to be mainly postsynaptic, but some immunoreactivity could also be discerned within the HRP-labeled presynaptic boutons. Because the wingless ligand may be encountered within the cell that secretes it, at the surface of the cell that binds it, and internalized into the cell with its receptors, the immunolocalization of wingless strongly indicates a role for the ligand at this synapse but does not identify the direction of signaling.

Because we wanted to address which downstream molecules may participate in NMJ wingless signaling, we further examined the requisite wingless coreceptor arrow, an essential member of the canonical signaling pathway. We found arrow immunoreactivity at the synapse, largely colocalizing with the neuronal elements, although a localization to the postsynaptic side of the synapse cannot be excluded by this light-microscopic analysis, and additional puncta of immunoreactivity were present in muscle cytosol. Because arrow is essential for the canonical signaling pathway but has not yet been found in any alternative wingless signaling context, its presence at the synapse suggests a role for the canonical pathway or its local variant at the NMJ.

To visualize the cytoplasmic protein dishevelled, we took advantage of a GFP-tagged full-length version, which was introduced as a transgene in Drosophila under the control of its endogenous promoter (J. Axelrod, personal communication). The GFP-labeled dishevelled appears enriched at the synapse, and its distribution indicates that both presynaptic and postsynaptic expression is likely: some GFP signal clearly overlaps with postsyn- 

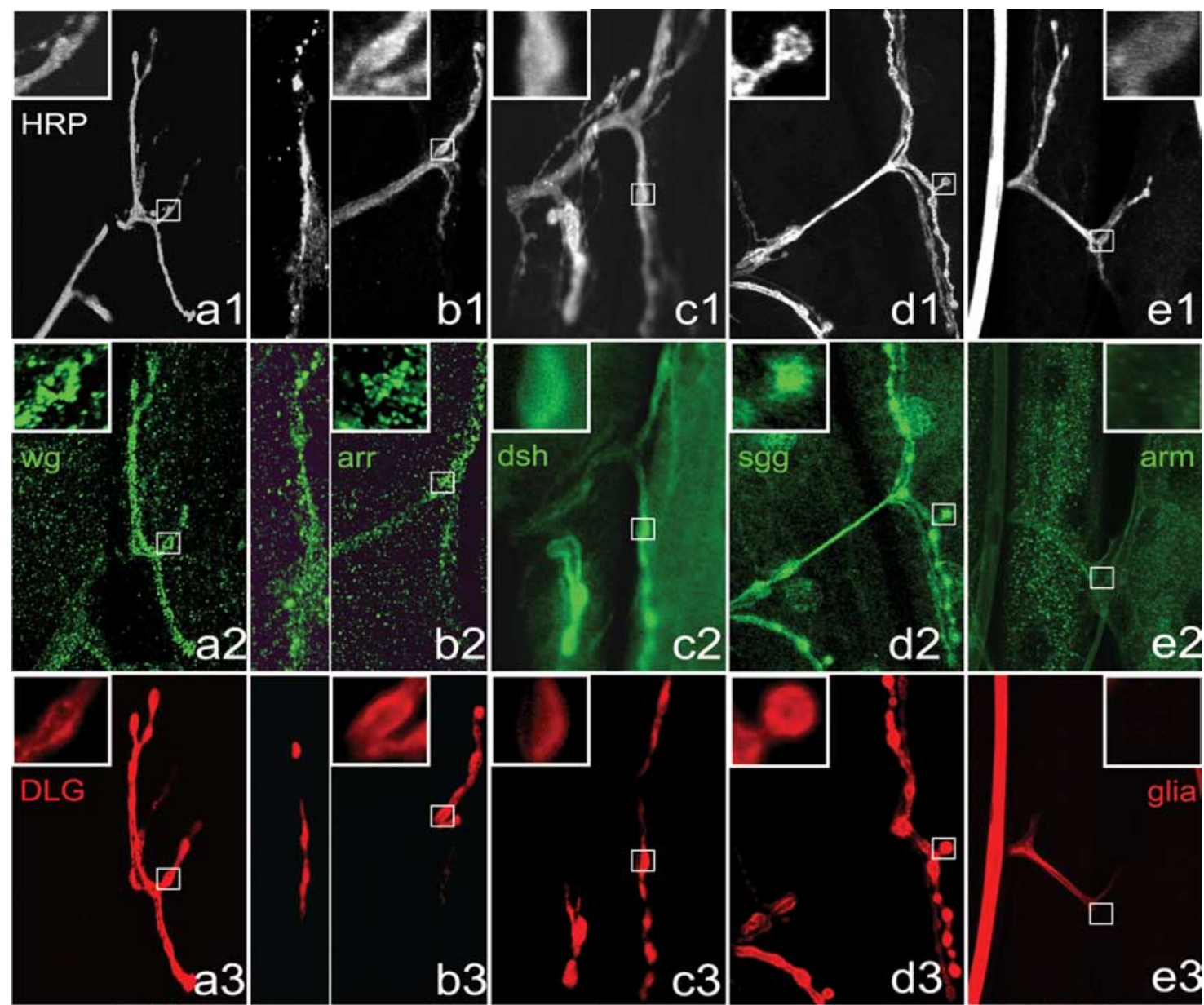

arm
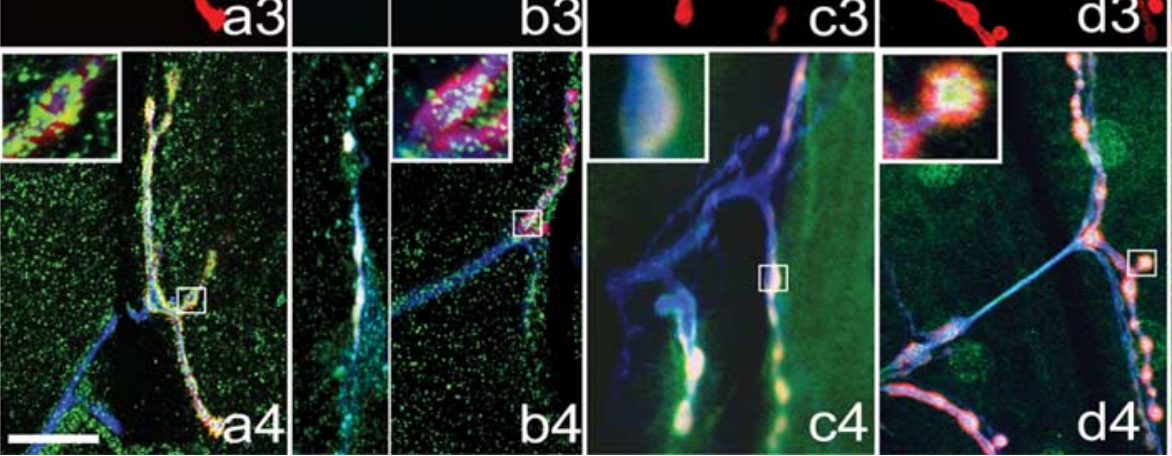

\section{d2}
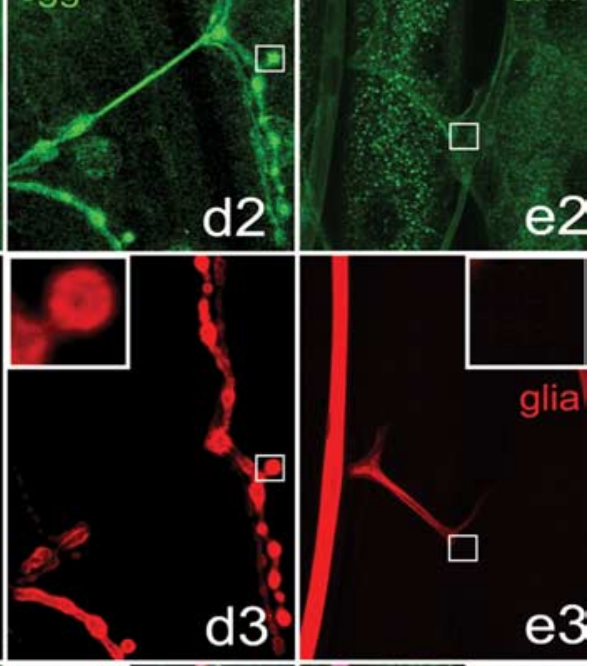

Figure 1. Canonical wg signaling components, but not $\beta$-catenin/armadillo, localize presynaptically and postsynaptically at the neuromuscular junction. Synapses on muscles $13 / 12$ were stained with anti-HRP (top row, white; bottom row, blue). Threefold magnifications of single boutons (marked with boxes) are shown as insets. $\boldsymbol{a}, \boldsymbol{b}$, Wild-type synapses stained with anti-Dlg (red) and either anti-wg ( $\boldsymbol{a}$, green) or anti-arr ( $\boldsymbol{b}$, green). Wg and arrow immunoreactivity show punctate distributions in the muscle and synaptic enrichment. Arrow is also detectable in the axon and is quite prominent presynaptically. c, A synapse expressing GFP-tagged dsh under its endogenous promoter (green) and stained with anti-Dlg (red). Dsh is enriched at the synapse and expressed in both the muscle and nerve. $\boldsymbol{d}$, A wild-type synapse stained with anti-DLG (red) and anti-GSK3/shaggy (green). Sgg is enriched at axons and boutons and is diffusely present in the muscle. $\boldsymbol{e}$, Anti-armadillo immunostaining (green) localizes to glia in the segmental nerve, marked by expression of UAS mCD8-GFP under control of the glia-Specific driver repo-GAL4 (red). Armadillo is also detected in puncta in the muscle, but is generally absent from muscle nuclei or synaptic regions. Scale bar: $15 \mu \mathrm{m}$; insets, $5 \mu \mathrm{m}$.

aptic Dlg staining, but it is also apparent within the presynaptic bouton and axon. Dishevelled is thus likely to act downstream of arrow, probably on both sides of the synapse. If these proteins represent the first steps of the canonical pathway, members of the $\beta$-catenin destruction complex should also be present, including shaggy. With antibodies to shaggy, we confirmed the observation of Franco et al. (2004) that shaggy is present at the synapse and in particular can be observed in the presynaptic nerve and endings (Fig. 1). Together, the colocalization of wingless, arrow, dishevelled, and shaggy provided evidence for the presence of canonical wingless signaling at the NMJ of Drosophila larvae.
Armadillo localizes to the nerve but is not enriched in synaptic areas

The classical canonical pathway controls gene expression via $\beta$-catenin/armadillo translocation to the nucleus. Immunolocalization of armadillo at the NMJ, however, revealed immunoreactivity in the axonal sheath but not at the synaptic endings (Fig. 1). The armadillo immunoreactivity did not appear to be neuronal but instead colocalized with a marker of glial membranes, as visualized by a fusion of CD8 to GFP expressed via the glial specific driver repo-GAL4. The absence of armadillo staining at the larval NMJ therefore suggested that wingless signaling might oc- 
cur via the local variant of the canonical pathway that is independent of $\beta$-catenin/ armadillo stabilization.

$w g$ and arrow mutants share similar neuromuscular junction defects

To determine whether the signaling proteins we localized to the NMJ indeed have a role in wingless signaling at this synapse, we inquired whether loss of function alleles for these proteins would mimic the reported $w g \mathrm{NMJ}$ phenotypes. Because the canonical wingless signaling pathway is crucial in early fly development, we could not determine the NMJ phenotypes of null alleles. Instead, we used either hypomorphic allelic combinations that survived until the third instar or dominant-negative constructs that could be selectively expressed in the neurons. To determine the function of arrow at this synapse, we therefore examined heterozygous third-instar larvae carrying one null and one hypomorphic allele $\left(\mathrm{arr}^{2} / \mathrm{arr}^{\mathrm{K} 08131}\right)$, a genotype that survives until the pupal stage. We compared those NMJs to those of a similarly viable combination of alleles of $w g$ $\left(w g^{C X 4} / w g^{t s}\right)$ and to larvae overexpressing wingless (UAS-wg). arrow (arr) mutant larvae indeed exhibited the reported $w g$ mutant phenotype with its markedly altered synaptic morphology (Fig. 2). In both arr and $w g$ mutant larvae, bouton numbers were significantly reduced compared with wt, and the number of enlarged $(>5 \mu \mathrm{m})$ and irregularly shaped boutons was significantly increased, as previously reported for $w g$ (Packard et al., 2002). NMJ formation is also sensitive to $w g$ overexpression; Packard et al. (2002) found that overexpression of a wg-GFP transgene in neurons caused an increase in bouton number and the appearance of small satellite boutons on short branches protruding from the major neurites. We confirmed these results with an untagged $w g$ transgene (Fig. 2).

\section{The formation of futsch-associated microtubule loops is} disrupted in arrow mutants

The microtubule-associated protein futsch is essential for synaptic growth and regulation of the microtubule cytoskeleton. Regular synaptic growth requires futsch to associate with loops of bundled microtubules within a subset of the boutons at the NMJ. If futsch is absent, synaptic growth and morphology are severely impaired (Roos et al., 2000). The formation of these futsch loops is diminished in $w g$ mutant larvae; within many of the mutant boutons, futsch shows an unbundled, splayed, or punctate distribution instead (Packard et al., 2002). We therefore asked whether futsch distribution was similarly altered in arr mutants (Fig. 3). In both wild-type and arr mutants, futsch was associated with microtubule bundles along the axon and in many of the normally shaped arr boutons, loops of microtubules remained. However, in the enlarged arr boutons of $>5 \mu \mathrm{m}$ diameter, futsch appeared unbundled and punctate. This resulted in a net decrease in the number of futsch loops at arr mutant terminals and an in- crease in the number of boutons with punctuate or unbundled futsch immunoreactivity (Fig. 4). We costained arr mutant larvae with antibodies to $\alpha$-tubulin to visualize the microtubules (data not shown) and found that boutons filled with unbundled futsch lacked microtubule loops, as expected. Thus, decreasing the arrow coreceptor activity for canonical wingless signaling caused cytoskeletal abnormalities at the synaptic endings.

To characterize further the large and irregular boutons observed at arr mutant NMJs, we examined the distribution of mitochondria by expressing in neurons a modified GFP that is localized to mitochondria (Pilling et al., 2006). We did not find any difference in the distribution of mitochondria between wt and $a r r$ mutant axons or in the arr synaptic boutons that were $<5 \mu \mathrm{m}$ in diameter. In the enlarged and irregularly shaped arr boutons, however, mitochondria were sparse (Fig. $3 b, f$ ). Thus, the local disorganization of microtubules in these large boutons was not reflective of a general dysfunction of microtubules that would prevent axonal transport of mitochondria, although it was paralleled by a local loss of mitochondria. The enlarged boutons, however, nonetheless contained active zones [as indicated by immunostaining with the marker antibody bruchpilot (nc82)] and were adjacent to postsynaptic concentrations of glutamate receptors (Fig. $3 c, g$ ), similar to $w t$ boutons.

Rescue of the arrow phenotype indicates both presynaptic and postsynaptic functions of arrow

Although previous studies of wingless signaling at NMJs have described a postsynaptic signaling pathway (Mathew et al., 2005), 

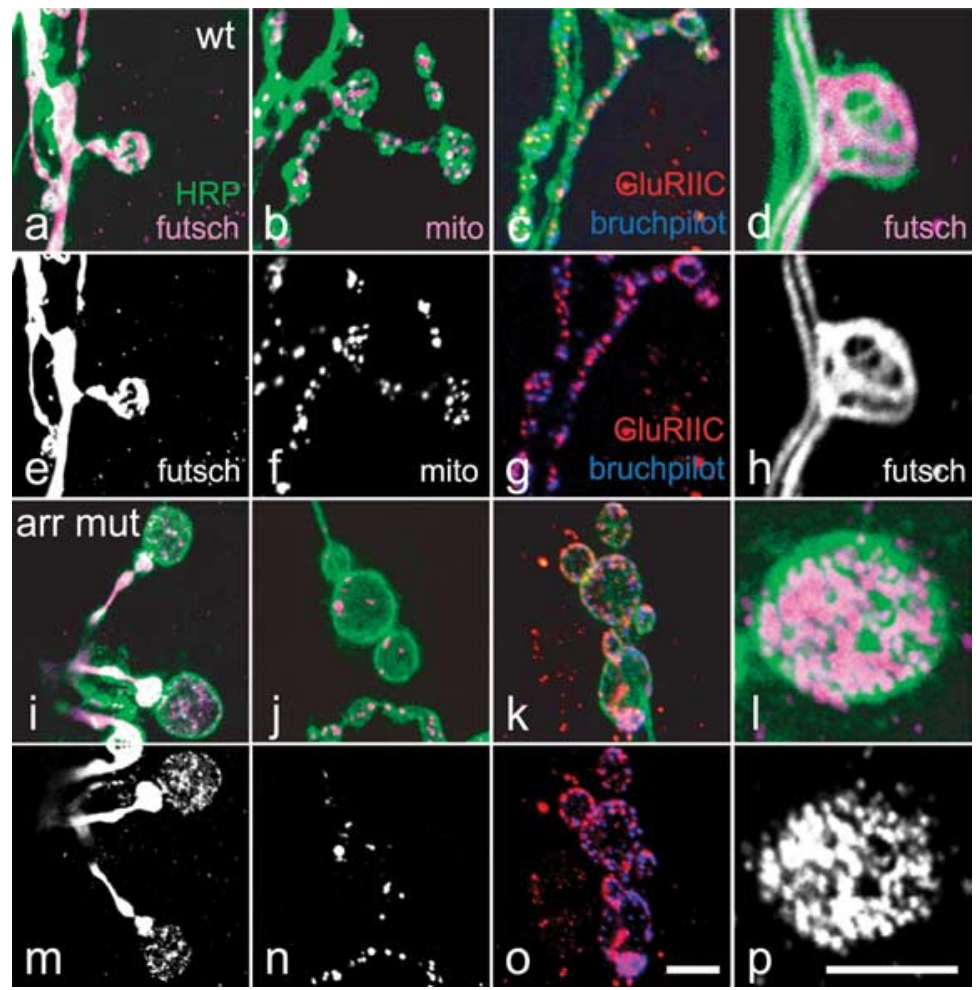

Figure 3. Abnormal microtubule structures in enlarged arr boutons. $\boldsymbol{a}-\boldsymbol{p}$, Wild-type $(\boldsymbol{a}-\boldsymbol{h})$ and arr $^{2} /$ arr $^{K 08131}$ mutant (mut; $\boldsymbol{i}-\boldsymbol{p}$ ) synapses immunostained with anti-HRP (green) and for presynaptic and postsynaptic proteins as follows: futsch (magenta), mito-GFP (magenta), GluRIIC (red), and bruchpilot (blue). Individual single channels are also shown in gray-scale below ( $\boldsymbol{e}-\boldsymbol{h}$ $\boldsymbol{m}-\boldsymbol{p})$. Anti-futsch immunoreactivity $(\boldsymbol{a}, \boldsymbol{d}, \boldsymbol{i}, \boldsymbol{I}$, magenta; $\boldsymbol{e}, \boldsymbol{h}, \boldsymbol{m}, \boldsymbol{p}$, white) reveals the altered microtubule structure of arr mutant boutons. Although microtubules appear normally bundled within the axons, in the enlarged ( $>5 \mu \mathrm{m}$ ) boutons of arr mutants, diffuse punctuate labeling replaces the normal bundled and looped structures. Neuronal mitochondria, labeled with GFP $(\boldsymbol{b}, \boldsymbol{j}$, magenta; $\boldsymbol{f}, \boldsymbol{n}$, white), are present at arr mutant synapses, but scarce in those mutant boutons that are abnormally large. $\boldsymbol{c}, \boldsymbol{g}, \boldsymbol{k}, \boldsymbol{0}$, Monoclonal antibody bruchpilot (blue) and anti-GluRIIC (red) indicate that active zones and postsynaptic receptor clusters are present at mutant synapses, with a distribution similar to wild type. Scale bars: $\mathbf{o}$ (for $\boldsymbol{a}-\boldsymbol{c}, \boldsymbol{e}-\boldsymbol{g}, \boldsymbol{i}-\boldsymbol{k}, \boldsymbol{m}-\boldsymbol{0}), \boldsymbol{p}($ for $\boldsymbol{d}, \boldsymbol{h}, \boldsymbol{I}, \boldsymbol{p}), 5 \mu \mathrm{m}$.

the $w g$ phenotype entails changes in the presynaptic cytoskeleton. Moreover, immunolocalization of the components of the canonical pathway (Fig. 1) suggested that wingless may also signal directly to presynaptic terminals. To examine the potential relative contributions of the arrow-dependent pathways presynaptically and postsynaptically, we attempted to rescue the arr phenotype with expression of a UAS-arr transgene in either neurons or muscle by means of the elav-GAL4 and MHC-GAL4 drivers. Neuronally expressed arrow fully rescued most of the NMJ phenotypes (Fig. 4) and in some regards caused gain-of-function phenotypes. Thus, the number of boutons per synapse was not only restored but significantly increased ( $p<0.05$ ), and the number of boutons $>5 \mu \mathrm{m}$ was reduced to normal levels. Similarly, the number of boutons with punctuate, unbundled futsch and the number with detectable microtubule loops were both restored to wildtype levels. The number of satellite boutons also increased, as was observed with wingless overexpression. Presynaptic overexpression in a wild-type background also increased the number of boutons, satellite boutons, and boutons with microtubule loops, consistent with an increase in presynaptic wg signaling (data not shown). Thus, the arr phenotype is most likely attributable to loss of arr function, and loss of neuronal arrow signaling is critical for the morphological and cytoskeletal defects. Notably, however, muscle expression of arrow also significantly rescued these phenotypes (Fig. 4). Arrow expression in muscle restored the number of boutons to wild-type levels and induced numbers of satellites similar to those seen with presynaptic expression. However, distribution (Fig. 5). The function of TCF/pangolin (pan), the transcription factor downstream of the canonical pathway that mediates its nuclear effects, can also be suppressed by a dominant-negative construct $\left(p a n^{D N}\right)$. Expression of this construct in muscle, however, produced a severe developmental phenotype characterized by thin muscle strands with an irregular surface (supplemental Fig. S1, available at www. jneurosci.org as supplemental material). Bouton formation on these muscles was severely affected; still, loops of microtubules with futsch immunoreactivity remained and even increased in number, possibly as a secondary effect of decreased bouton growth. Thus, although the gross abnormalities of the muscle fibers made a quantitative comparison to arr and $\mathrm{wg}$ phenotypes impossible, the phenotypes were sufficiently distinct as to suggest that pangolin in the muscle, like dishevelled, was not directly responsible for the $w g$ phenotype in the boutons.

\section{Futsch-loop formation in boutons requires presynaptic} dishevelled and shaggy

In contrast to our findings with muscle expression, $d s h^{D I X}$ expression in neurons via elav-GAL4 caused a strong NMJ phenotype resembling that of $w g$ and $\operatorname{arr}$ (Fig. 5). Bouton count was reduced, and enlarged and irregularly shaped boutons were more frequent. The number of futsch-labeled loops was decreased, and the number of boutons containing unbundled futsch (arrows) was significantly increased $(p<0.0005)$. Because the 
dishevelled-containing destruction complex antagonizes shaggy, disruption of $d s h$ function is expected to be equivalent to a gain of shaggy ( $s g g$ ) function, whereas sgg loss-of-function mutants should yield the opposite phenotype. Indeed, Franco et al. (2004) found a significant increase in the number of boutons, satellites, and futsch loops for the presynaptic suppression of sgg function with a dominant-negative construct. In our hands, when $s g g^{D N}$ was driven in neurons with elav-GAL4, the number of satellite boutons and futsch loops was increased, but the number of boutons, although slightly greater than in wild type, did not reach statistical significance.

Armadillo and pangolin do not regulate microtubule and bouton morphology We next tested whether the canonical wingless pathway signals in a transcription-dependent or independent manner by examining mutations of arm and pan. Wingless signaling in the canonical pathway normally inhibits phosphorylation of armadillo by shaggy and thereby allows the dephosphorylated form to accumulate and translocate to the nucleus, where, together with pangolin, it activates target genes. Therefore inhibition of either armadillo or pangolin is predicted to mimic the phenotype of $w g$ if this pathway controls synaptogenesis. To this end, we expressed in the nervous system a dominant-negative form of pangolin, to inhibit armadillo signaling and also a constitutively active form of armadillo that should mimic the $w g$ overexpression phenotype (Fig. 5). We did not observe any differences in synapse formation and morphology in these animals compared with wild type. Expression of these constructs in muscle also failed to produce a phenotype like that of $w g$, although we found the constitutively active armadillo enriched within the muscle nuclei (supplemental Fig. S2, available at www.jneurosci.org as supplemental material). Wingless was therefore more likely to be acting by local actions of shaggy in nerve endings, rather than via a transcriptional pathway.

To test this hypothesis further, we examined whether the constitutively active armadillo might overcome deficiency in $d s h$ function (Fig. 5n). Thus, dominant-negative $d s h$ and constitutively active arm were simultaneously expressed in the nervous system (Fig. 5g). The phenotype of these flies closely resembled that of a $w g$ mutant fly or a fly expressing the dominant-negative $d s h$ alone. Thus, the engineered activation of armadillo could not circumvent the requirement of $d s h$ function. In a converse experiment, we expressed a dominant-negative sgg together with a dominant-negative pan (Fig. 5h). In these flies, the canonical wingless pathway should be stimulated by the suppression of shaggy function, but its transcriptional consequences would be blocked. The phenotype of these flies closely resembled the phenotype of $w g$ overexpression or the dominant-negative form of shaggy alone. Thus, activation of the cytoplasmic components of the canonical pathway in the presynaptic neurons was sufficient

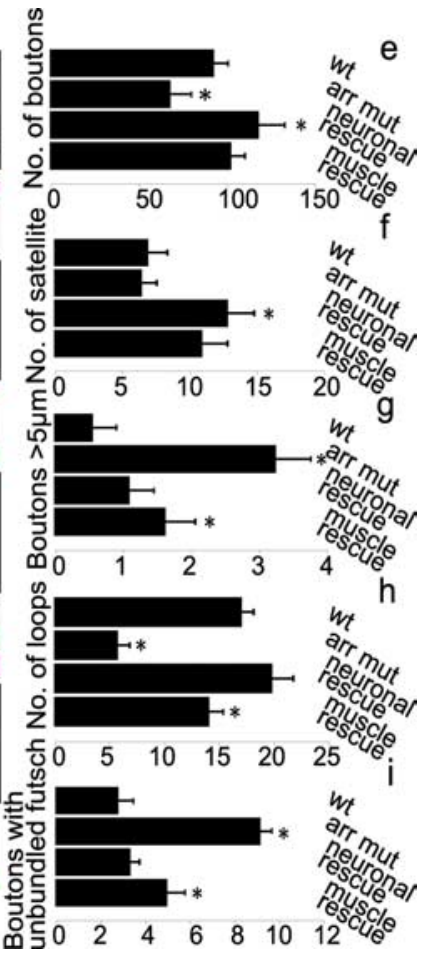

Figure 4. Neuronally expressed arrow rescues the NMJ phenotypes of arr. Synapses of segment $A 3$, muscles $7 / 6$, were costained with anti-HRP (white; green in insets) and anti-futsch (magenta). $\boldsymbol{a}$, Wild type. $\boldsymbol{b}$, arr $^{2} / \mathrm{arr}^{\mathrm{K} 08131}$ mutant (mut). $\boldsymbol{c}$, Rescue (resc) of arr phenotype by neuronal expression of arrow in genotype $\operatorname{arr}^{2} / \operatorname{arr}^{K 08131}$;elan-GAL4/UAS-arr. $\boldsymbol{d}$, Partial rescue of arr of arrow in muscles restored normal numbers of boutons, but some oversized boutons remained, and the microtubule structure was not completely restored. $n=12$ wild-type larvae (21 NMJs), 15 arr larvae (28 NMJs), 16 neuronal rescue larvae (31 NMJs), and

to mimic wg function in controlling bouton number and structure, and the number of futsch-immunoreactive microtubule loops, apparently in an armadillo/TCF-independent manner.

The mammalian homolog of shaggy, GSK $3 \beta$, has been reported to phosphorylate the mammalian homolog of futsch, MAP1B (Gordon-Weeks and Fischer, 2000), and a similar phosphorylation occurs in Drosophila (Gögel et al., 2006). We therefore examined whether the presynaptic wingless pathway at the fly NMJ acts via futsch to regulate bouton number and structure, or whether the alterations in futsch loops are incidental to the other phenotypes. To this end, we activated the pathway by overexpression of either $w g$ or dominant-negative $s g g$ in neurons in the context of larvae either heterozygous or homozygous for fut$\operatorname{sch}^{K 68}$ (supplemental Fig. S3, available at www.jneurosci.org as supplemental material). Consistent with a model in which this pathway is mediated by phosphorylation of futsch, the increase in bouton number and satellite boutons did not occur in the futsch mutant backgrounds. Moreover, the phenotype of futsch homozygotes, a decrease in bouton number and an increase in boutons $>5 \mu \mathrm{m}$, could not be overcome by expression of either $w g$ or dominant-negative sgg. These genetic interactions imply that futsch is a requisite target of the wingless pathway for controlling development of this synapse.

\section{Discussion}

We have examined the Drosophila neuromuscular junction as a model system in which to explore wingless signaling mechanisms 

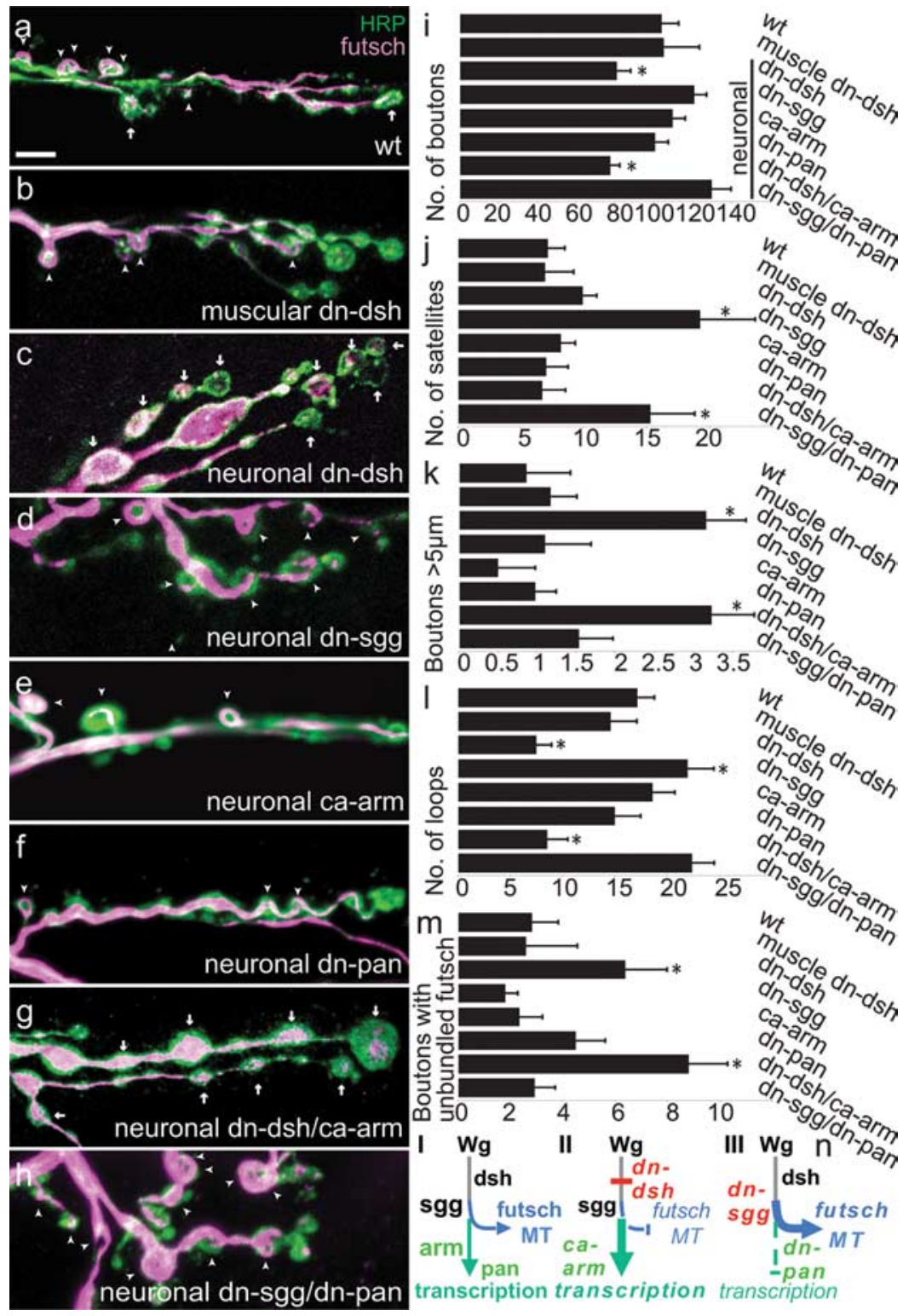

140
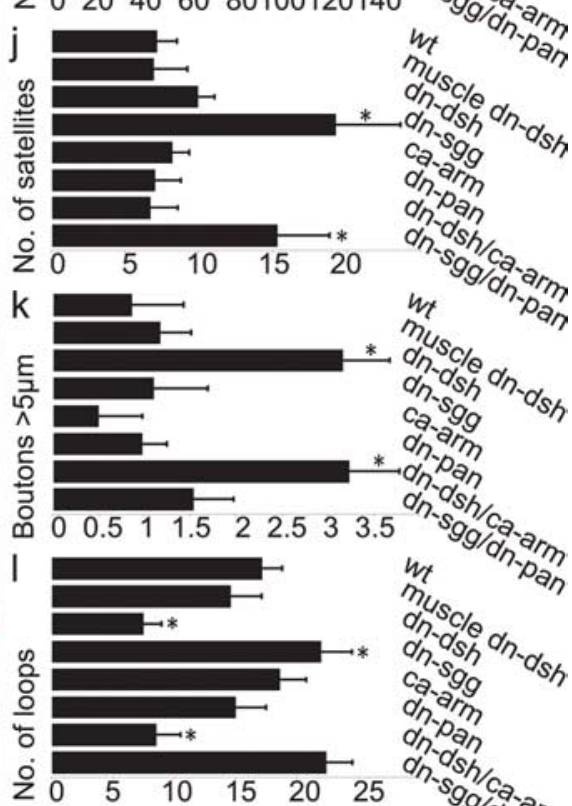

ararm

dran

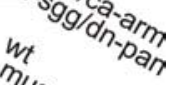

$\mathrm{m}_{4 \mathrm{~s}}$

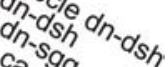

a.

di $a^{2}$ m

0

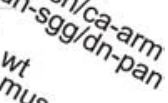

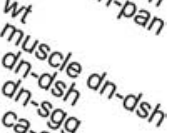

a. 2,

do

oh
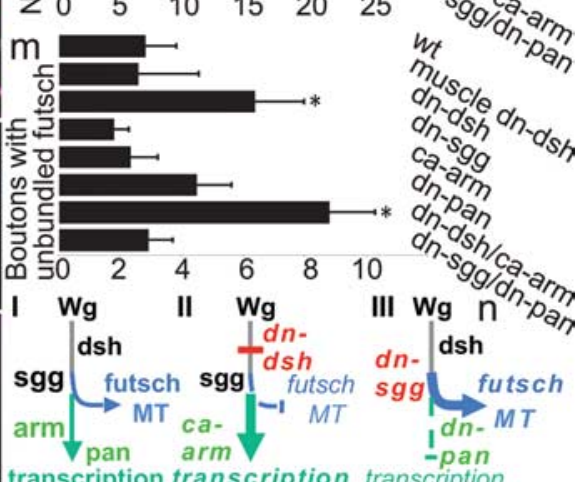

Figure 5. Microtubule-loop formation in boutons requires canonical wg signaling components presynaptically, but is independent of armadillo and pangolin. $\boldsymbol{a}-\boldsymbol{h}$, Muscles 7/6 synapses stained with anti-HRP (green) and anti-futsch (magenta). Futsch-immunoreactive microtubule loops (arrowheads) and boutons with unbundled futsch (arrows) are marked. $\boldsymbol{a}$, Wild type. $\boldsymbol{b}$, UAS-dsh ${ }^{D I X} ; M H C-G A L 4$. Normal synaptic architecture persists when the dominant-negative construct $d s h^{D I X}$ is expressed in the muscles. c, UAS-dsh ${ }^{D I X}$;elav-GAL4. Neuronal expression of $d s h^{D I X}$ causes an arr-like phenotype with enlarged boutons and fewer microtubule loops. $\boldsymbol{d}$, UAS-sgg ${ }^{A 82 T}$;elav-GAL4. Neuronal expression of a dominant-negative construct of $\mathrm{sg} g$ increases the number of satellite boutons. e, UAS-arm ${ }^{510}$;elav-GAL4.f, UAS-pan ${ }^{\Delta N}$; elav-GAL4. Neither expression of constitutively activated armadillo nor expression of dominant-negative pangolin resulted in an arr-like synaptic phenotype. $g$, UAS-dsh ${ }^{\text {DIX }} /$ UAS-arm ${ }^{510}$;elav-GAL4. Simultaneously activating armadillo and inhibiting dishevelled in neurons caused the arr-like phenotype. $\boldsymbol{h}$, UAS-sgg ${ }^{A 82 T} /$ UAS-pan ${ }^{\Delta N}$;elav-GAL4. Simultaneously inhibiting shaggy and armadillo (via a dominant-negative pangolin) in neurons produces the sgg dominant-negative phenotype. $\boldsymbol{i}-\boldsymbol{m}$, For the genotypes in $\boldsymbol{a}-\boldsymbol{h}$, quantification of bouton number $(\boldsymbol{i})$, satellite bouton number $(\boldsymbol{j})$, number of boutons $>5 \mu \mathrm{m}(\boldsymbol{k})$, number of boutons with futsch-immunoreactive loops $(\boldsymbol{I})$, and number of boutons with unbundled futsch $(\boldsymbol{m}) . \boldsymbol{n}, \mathrm{I}$, Diagram of the wg signaling pathway showing the two possible branches downstream of the wgdependent inhibition of shaggy: activation of transcription in the nucleus via armadillo and pangolin (green) or local effects of shaggy on microtubule structure (blue). II, III, IIlustration of the genotypes in $\boldsymbol{g}$ and $\boldsymbol{h}$ as a means to discriminate between these branches. In II, UAS $d n-d s h^{D I X}$ inhibits the regulation of shaggy, but a potential transcriptional branch can be activated by UAS ca-arm ${ }^{S 10}$ as in $\boldsymbol{g}$. In III, UAS dn-sgg ${ }^{A 82 T} /$ UAS dn-pan ${ }^{\Delta N}$ leads to the opposite effect of II, blocking the transcriptional branch but activating earlier elements of the wg pathway, including the inhibition of sgg. $n=20$ wild-type larvae (34 NMJs), 11 MHC-Gal4 UAS dn-dsh ${ }^{\text {DIX }}$ larvae (18 NMJs), 12 elav-Gal4, UAS dn-dsh ${ }^{\text {DIX }}$ larvae (22 NMJs), 12 elav-Gal4, UAS dn-sgg ${ }^{A 82 T}$ larvae (21 NMJs), 10 elav-Gal4, UAS ca-arm ${ }^{\text {S10 }}$ larvae (19 NMJs), 13 elav-Gal4, UAS dn-pan ${ }^{\Delta N}$ larvae (22

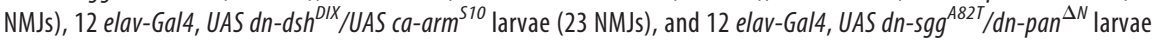
(20 NMJs). *Statistically different from wild type $(p<0.05)$. Scale bar, $20 \mu \mathrm{m}$. in synapse development. We found that proteins of the canonical wingless signaling pathway participate in the assembly of this synapse. Crucial components of the pathway are the canonical wingless coreceptor arrow, dishevelled, and the glycogen synthase kinase shaggy. We hypothesize, however, that this pathway branches away from the canonical signaling pathway that involves armadillo translocation to the nucleus and transcriptional control and, instead, involves local actions of shaggy. Shaggy acts, at least in part, by regulating the structure of the microtubule cytoskeleton, probably via the microtubule-associated protein futsch.

At the Drosophila NMJ, wingless promotes synaptic growth and differentiation (Packard et al., 2002). Lack of wingless reduces the number of boutons, some of which appear unevenly shaped and enlarged. To date, only a postsynaptic signaling pathway had been described at the NMJ (Mathew et al., 2005). This novel pathway entails the translocation of the cleaved $\mathrm{C}$ terminus of the frizzled-2 receptor into muscle nuclei. The scaffolding protein glutamate receptor-interacting protein (GRIP) supports the process (Ataman et al., 2006). Here, we demonstrate that wingless also acts on the presynaptic terminals and that the presynaptic and postsynaptic signaling cascades are distinct.

Proteins in the canonical wingless pathway regulate NMJ development An initial indication that the canonical pathway may function in addition to the frizzled-2 cleavage pathway came from the immunocytochemical determination that some pathway components were enriched at the NMJ. In particular, we found that arrow, dishevelled, and shaggy were concentrated at the synapse. The developmental significance of these components was determined by genetic manipulation. Those manipulations that blocked transduction through the canonical pathway mimicked the synaptic phenotype of $w g$ itself. Thus, mutations in arr, expression of a dominant-negative form of dishevelled, and overexpression of shaggy all gave rise to the phenotypes observed in wg: a decrease in bouton number, the appearance of boutons of $>5 \mu \mathrm{m}$ diameter, a decrease in the number of boutons with futschlabeled microtubule loops, and an increase in the number of boutons with diffuse or unbundled futsch. Notably, the dominant-negative form of dishevelled that we used $\left(d s h^{D I X}\right)$ is thought to interfere only with the interactions of dishev- 
elled with the canonical pathway (Axelrod et al., 1998). Furthermore, two manipulations that enhanced signaling through the canonical pathway mimicked the phenotype of overexpressed wingless. Thus overexpression of arrow in neurons caused bouton number to increase significantly, and overexpression of dominant-negative shaggy increased the number of satellite boutons and the number of boutons with futsch loops.

\section{Arrow, dishevelled, and shaggy transduce wingless signals presynaptically}

Because the $w g$ phenotype entails changes in the anatomy and internal cytoskeleton of the nerve endings, the development of the synapse must either entail a retrograde signal from the postsynaptic frizzled-2 pathway back to the boutons or an additional presynaptic pathway. The immunocytochemical localization of frizzled receptors to both sides of the synapse (Packard et al., 2002) as well as the localization of arrow, dishevelled, and shaggy (Fig. 1) suggested that these proteins may indeed be neuronal. Shaggy immunofluorescence in particular appeared to be within the boutons themselves (Fig. 1) (Franco et al., 2004). To address this matter more directly, transgenes were selectively expressed presynaptically or postsynaptically to determine on which side the canonical pathway was acting. Because of the potential redundancy of frizzled and frizzled-2 as receptors for wingless, we focused instead on downstream components. Disrupting the canonical pathway with $d s h^{D I X}$ expression was effective in producing a $w g$-like phenotype only when $d s h^{D I X}$ was expressed in neurons, but not in the muscle. The ineffectiveness of this transgene in the muscle suggests that the canonical pathway is not required postsynaptically. Similarly, the dominantnegative form of shaggy mimicked wingless overexpression when expressed presynaptically, and arrow expression presynaptically fully rescued the phenotypes of arr mutants. Each of these manipulations confirmed the functional importance of these canonical components in the neurons.

Notably, expression of arrow in muscle cells could also rescue much of the arr mutant phenotype; proper bouton number was restored by postsynaptic expression, and microtubule phenotypes were partially restored. Although heretofore arrow has only been shown to act in the canonical pathway, such a function in the muscle appears unlikely because of the ineffectiveness of $d s h$ $D I X$ expression in muscle cells. It is therefore tempting to speculate that arrow will also serve as a coreceptor for frizzled-2 in the pathway that leads to nuclear translocation of the frizzled-2 C terminus.

\section{Bidirectional signaling by wingless at the Drosophila NMJ}

The elucidation of a presynaptic pathway for wingless signaling at the NMJ raises the question of the respective roles of this pathway and the previously described postsynaptic pathway. In some regards, they are likely to act in concert. For example, postsynaptic rescue of frizzled-2 mutant phenotypes restored bouton numbers in frizzled-2 mutants. Because we also see evidence for presynaptic control of bouton number by the canonical pathway, it is likely that distinct but parallel wingless pathways on either side of the synapse participate in regulating this aspect of development. This is also consistent with our observation that bouton number in $\mathrm{arr}$ mutants can be rescued by either presynaptic or postsynaptic expression of arrow. On the other hand, only presynaptic expression of arrow could properly rescue the futsch/microtubule phenotypes, and we therefore propose that the regulation of futsch loop formation is predominantly presynaptic. In general, the presynaptic pathway appears to have a very potent effect on synapse

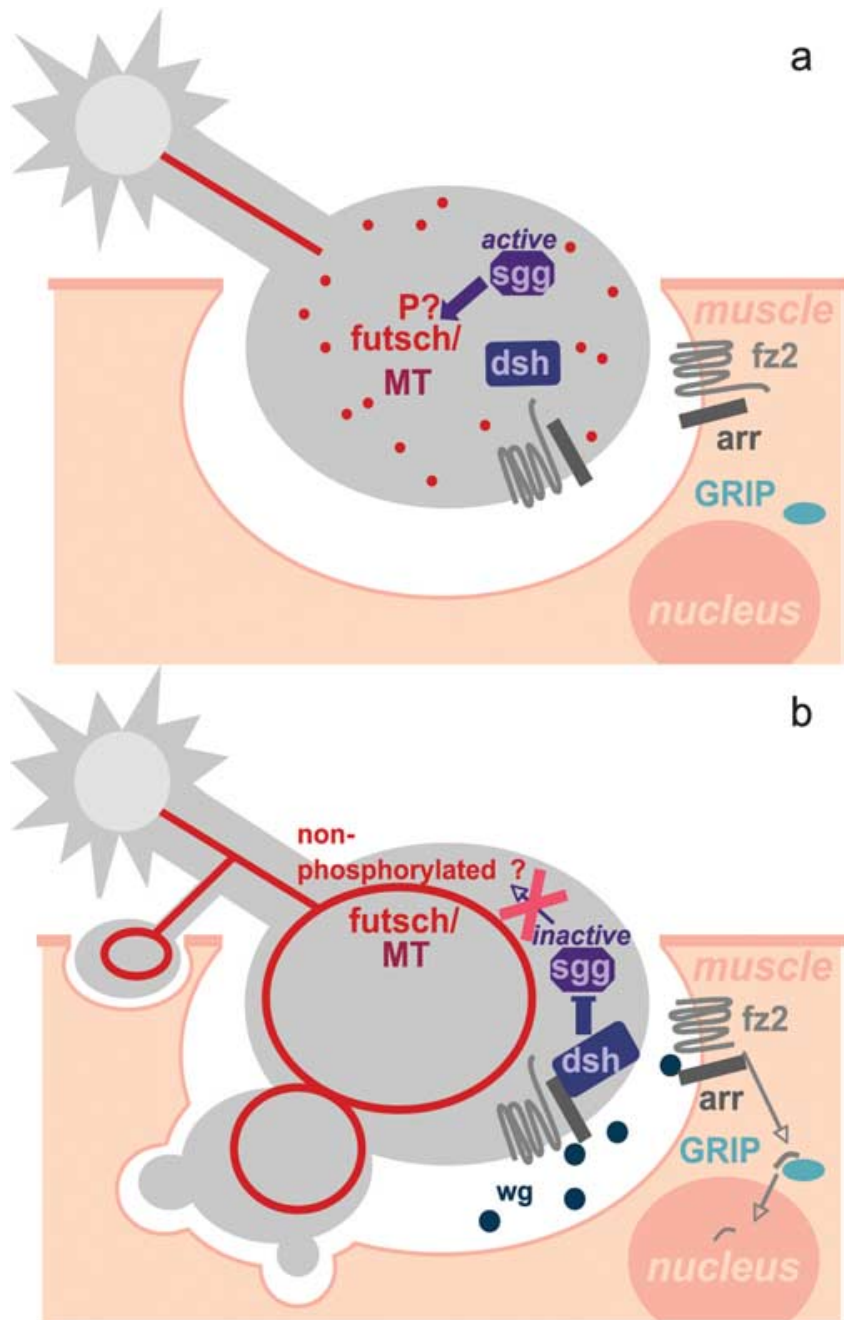

Figure 6. A model for wingless actions presynaptically and postsynaptically at the NMJ of Drosophila. $\boldsymbol{a}$, Without a wingless signal, presynaptic dishevelled remains in an inactive state, and shaggy remains active, thereby preventing assembly of microtubule loops, probably by phosphorylation of futsch. Terminals therefore contain punctate futsch and depolymerized or disordered microtubules. The formation of additional boutons is blocked. $\boldsymbol{b}$, Wingless signaling at the Drosophila NMJ activates two different intracellular cascades. Postsynaptically, the C-terminal cleavage of the receptor frizzled2 takes place, possibly after wg binds to its receptorcoreceptor complex frizzled2/arrow. The $C$ terminus translocates to the muscle nucleus in a process requiring GRIP (Mathew et al., 2005; Ataman et al., 2006). At the presynaptic arbor, wingless binds to the frizzled2/arrow complex and activates dishevelled. The glycogen synthase kinase shaggy is thereby blocked, which permits futsch to promote the organization of microtubules into loops, the budding of new boutons, and synaptic growth.

development in that selective manipulation of $d s h$, sgg, or arr just in the nervous system produced phenotypes as severe as those evoked by the $w g$ mutations themselves.

\section{Canonical wingless signaling affects the presynaptic microtubule cytoskeleton independent of transcriptional control}

Elements of the canonical pathway were observed at the synapse, but only to the level of shaggy. Armadillo, the Drosophila $\beta$-catenin, was not detectable in the nerve endings [as also reported by Packard et al. (2002)]. Nor was it observed in axons of the peripheral nerves or neuronal nuclei (supplemental Fig. S2, available at www.jneurosci.org as supplemental material). Instead, it was detected in the glial sheath at the synapse and in the neuropil of the CNS. The canonical wingless pathway, which 
controls gene expression, leads to the translocation of armadillo to the nucleus in response to wingless signaling. Thus, a constitutively active form of armadillo translocates to the nuclei of epithelial and muscle cells (supplemental Fig. S2, available at www.jneurosci.org as supplemental material) (Tolwinski and Wieschaus, 2001). When we expressed the constitutively armadillo in neurons, however, we could not mimic wg overexpression, nor could we mimic wg mutant phenotypes by blocking armadillo function with dominant-negative pangolin. In addition, dominant-negative pangolin could not block activation of the pathway by dominant-negative shaggy, and constitutively active armadillo could not override the upstream block of the canonical pathway by $d s h^{D I X}$. Thus, the synaptic form of the canonical pathway appears to function only to the level of shaggy.

We therefore favor a model, like that described in mammalian cerebellar synapses (Hall et al., 2000), in which GSK3 $\beta$ can act locally to modify the cytoskeleton independently of $\beta$-catenin/ TCF signaling and transcriptional regulation. In this model, wingless signals lead directly to the polymerization and stabilization of microtubules (Fig. 6), via phosphorylation of futsch by shaggy. The genetic interactions of $w g$ overexpression and presynaptic expression of dominant-negative sgg (supplemental Fig. S3, available at www.jneurosci.org as supplemental material) supported this model. Mammalian GSK3 $\beta$ phosphorylates MAP1B, the mammalian homolog of futsch (Gordon-Weeks and Fischer, 2000), and this event appears to be under the control of Wnt proteins. Wnt7a and Wnt7b signaling reduces GSK3 $\beta$ activity and MAP1B phosphorylation (Ciani et al., 2004). Indeed, shaggy has been shown to phosphorylate serine 4867 of futsch (Gögel et al., 2006). Because futsch is known to have an important role in stabilizing the structure of the synaptic microtubules and thereby to regulate bouton growth (Roos et al., 2000; Zhang et al., 2001), this local variant of the canonical pathway (Fig. 6) appears to be sufficient to explain the presynaptic actions of wingless.

Previously, this unique additional branch of the canonical Wnt pathway was described exclusively in mammalian neuronal culture (Ciani et al., 2004). Here we provide genetic data demonstrating a similar pathway for NMJ differentiation in Drosophila larvae. It appears that this presynaptic Wnt/wingless pathway represents a specialized and conserved branch. It promotes axonal growth cone and synaptic differentiation in a local and $\beta$-catenin/transcription-independent manner. Further studies on different types of synapses in different species may elucidate whether this specialized canonical branch is widely used in synaptic development.

\section{References}

Ahmad-Annuar A, Ciani L, Simeonidis I, Herreros J, Fredj NB, Rosso SB, Hall A, Brickley S, Salinas PC (2006) Signaling across the synapse: a role for Wnt and Dishevelled in presynaptic assembly and neurotransmitter release. J Cell Biol 174:127-139.

Ataman B, Ashley J, Gorczyca D, Gorczyca M, Mathew D, Wichmann C, Sigrist SJ, Budnik V (2006) Nuclear trafficking of Drosophila Frizzled-2 during synapse development requires the PDZ protein dGRIP. Proc Natl Acad Sci U S A 103:7841-7846.

Axelrod JD, Miller JR, Shulman JM, Moon RT, Perrimon N (1998) Differential recruitment of Dishevelled provides signaling specificity in the planar cell polarity and Wingless signaling pathways. Genes Dev $12: 2610-2622$.

Cho KO, Hunt CA, Kennedy MB (1992) The rat brain postsynaptic density fraction contains a homolog of the Drosophila discs-large tumor suppressor protein. Neuron 9:929-942.

Ciani L, Krylova O, Smalley MJ, Dale TC, Salinas PC (2004) A divergent canonical WNT signalling pathway regulates microtubule dynamics: dishevelled signals locally to stabilize microtubules. J Cell Biol 164:243-253.

Dean C, Dresbach T (2006) Neuroligins and neurexins: linking cell adhesion, synapse formation and cognitive function. Trends Neurosci 29:21-29.

Elia LP, Yamamoto M, Zang K, Reichardt LF (2006) p120 catenin regulates dendritic spine and synapse development through Rho-family GTPases and cadherins. Neuron 51:43-56.

Estes PS, Jackson TC, Stimson DT, Sanyal S, Kelly LE, Ramaswami M (2003) Functional dissection of a eukaryotic dicistronic gene: transgenic stonedB, but not stonedA, restores normal synaptic properties to Drosophila stoned mutants. Genetics 165:185-196.

Franco B, Bogdanik L, Bobinnec Y, Debec A, Bockaert J, Parmentier ML, Grau Y (2004) Shaggy, the homolog of glycogen synthase kinase 3, controls neuromuscular junction growth in Drosophila. J Neurosci 24:6573-6577.

Gögel S, Wakefield S, Tear G, Klämbt C, Gordon-Weeks PR (2006) The Drosophila microtubule associated protein Futsch is phosphorylated by Shaggy/Zeste-white 3 at an homologous GSK3beta phosphorylation site in MAP1B. Mol Cell Neurosci 33:188-199.

Goold RG, Owen R, Gordon-Weeks PR (1999) Glycogen synthase kinase $3 \beta$ phosphorylation of microtubule-associated protein $1 \mathrm{~B}$ regulates the stability of microtubules in growth cones. J Cell Sci 112:3373-3384.

Gordon-Weeks PR, Fischer I (2000) MAP1B expression and microtubule stability in growing and regenerating axons. Microsc Res Tech 48:63-74.

Hall AC, Lucas FR, Salinas PC (2000) Axonal remodeling and synaptic differentiation in the cerebellum is regulated by WNT-7a signaling. Cell 100:525-535.

He X, Semenov M, Tamai K, Zeng X (2004) LDL receptor-related proteins 5 and 6 in Wnt/beta-catenin signaling: arrows point the way. Development 131:1663-1677.

Jan LY, Jan YN (1982) Antibodies to horseradish peroxidase as specific neuronal markers in Drosophila and in grasshopper embryos. Proc Natl Acad Sci U S A 79:2700-2704.

Krylova O, Messenger MJ, Salinas PC (2000) Dishevelled-1 regulates microtubule stability: a new function mediated by glycogen synthase kinase- $3 \beta$. J Cell Biol 151:83-94

Lee T, Luo L (1999) Mosaic analysis with a repressible cell marker for studies of gene function in neuronal morphogenesis. Neuron 22:451-461.

Logan CY, Nusse R (2004) The Wnt signaling pathway in development and disease. Annu Rev Cell Dev Biol 20:781-810.

Marrus SB, Portman SL, Allen MJ, Moffat KG, DiAntonio A (2004) Differential localization of glutamate receptor subunits at the Drosophila neuromuscular junction. J Neurosci 24:1406-1415.

Mathew D, Ataman B, Chen J, Zhang Y, Cumberledge S, Budnik V (2005) Wingless signaling at synapses is through cleavage and nuclear import of receptor DFrizzled2. Science 310:1344-1347.

Moon RT (2005) Wnt/beta-catenin pathway. Sci STKE 2005:cm1.

Packard M, Koo ES, Gorczyca M, Sharpe J, Cumberledge S, Budnik V (2002) The Drosophila wnt, wingless, provides an essential signal for pre- and postsynaptic differentiation. Cell 111:319-330.

Packard M, Mathew D, Budnik V (2003) Wnts and TGF beta in synaptogenesis: old friends signalling at new places. Nat Rev Neurosci 4:113-120.

Pilling AD, Horiuchi D, Lively CM, Saxton WM (2006) Kinesin-1 and dynein are the primary motors for fast transport of mitochondria in Drosophila motor axons. Mol Biol Cell 17:2057-2068.

Pinson KI, Brennan J, Monkley S, Avery BJ, Skarnes WC (2000) An LDLreceptor-related protein mediates Wnt signalling in mice. Nature 407:535-538.

Reichsman F, Smith L, Cumberledge S (1996) Glycosaminoglycans can modulate extracellular localization of the wingless protein and promote signal transduction. J Cell Biol 135:819-827.

Rives AF, Rochlin KM, Wehrli M, Schwartz SL, DiNardo S (2006) Endocytic trafficking of Wingless and its receptors, Arrow and DFrizzled-2, in the Drosophila wing. Dev Biol 293:268-283.

Roos J, Hummel T, Ng N, Klämbt C, Davis GW (2000) Drosophila Futsch regulates synaptic microtubule organization and is necessary for synaptic growth. Neuron 26:371-382.

Scheiffele P, Fan J, Choih J, Fetter R, Serafini T (2000) Neuroligin expressed in nonneuronal cells triggers presynaptic development in contacting axons. Cell 101:657-669. 
Speese SD, Budnik V (2007) Wnts: up-and-coming at the synapse. Trends Neurosci 30:268-275.

Stewart BA, Atwood HL, Renger JJ, Wang J, Wu CF (1994) Improved stability of Drosophila larval neuromuscular preparations in haemolymphlike physiological solutions. J Comp Physiol [A] 175:179-191.

Tamai K, Semenov M, Kato Y, Spokony R, Liu C, Katsuyama Y, Hess F, Saint-Jeannet JP, He X (2000) LDL-receptor-related proteins in Wnt signal transduction. Nature 407:530-535.

Tolwinski NS, Wieschaus E (2001) Armadillo nuclear import is regulated by cytoplasmic anchor Axin and nuclear anchor dTCF/Pan. Development 128:2107-2117.

Trivedi N, Marsh P, Goold RG, Wood-Kaczmar A, Gordon-Weeks PR (2005) Glycogen synthase kinase-3beta phosphorylation of MAP1B at Ser1260 and Thr1265 is spatially restricted to growing axons. J Cell Sci 118:993-1005.
Wallingford JB, Habas R (2005) The developmental biology of Dishevelled: an enigmatic protein governing cell fate and cell polarity. Development 132:4421-4436.

Wehrli M, Dougan ST, Caldwell K, O’Keefe L, Schwartz S, Vaizel-Ohayon D, Schejter E, Tomlinson A, DiNardo S (2000) arrow encodes an LDLreceptor-related protein essential for Wingless signalling. Nature 407:527-530.

Yao KM, White K (1994) Neural specificity of elav expression: defining a Drosophila promoter for directing expression to the nervous system. J Neurochem 63:41-51.

Zhang YQ, Bailey AM, Matthies HJ, Renden RB, Smith MA, Speese SD, Rubin GM, Broadie K (2001) Drosophila fragile X-related gene regulates the MAP1B homolog Futsch to control synaptic structure and function. Cell 107591-603. 